UNDERGRADUATE RESEARCH IN NATURAL AND CLINICAL SCIENCE AND TECHNOLOGY (URNCST) JOURNAL Read more URNCST Journal articles and submit your own today at: https://www.urncst.com

\title{
Scinapse 2018-2019 Undergraduate Science Case Competition: Cannabis, Harm or Health?
}

\author{
Dominic Cyr, BSc [1], Jamie Ghossein, BSc [1], Rashida Rajgara, MSc [1]* \\ [1] Faculty of Medicine, University of Ottawa, Ottawa, ON, Canada. \\ *Corresponding Author: rrajg025@uottawa.ca
}

\begin{abstract}
The Scinapse Undergraduate Science Case Competition (USCC) provides an opportunity for undergraduate students to experience the development of a novel research proposal. A case is presented to all participants and, using in-depth literature search (publications, reports, studies and published writings), students connect and pinpoint key elements allowing them to develop a hypothesis in support of the case in question. Participants also develop a methodology which will test the validity of their hypothesis. This year's case topic was inspired by the recent legalization of cannabis in Canada. This controversial topic provided an opportunity for participants to explore research proposals focused on either the health aspect of cannabis or the potential harm posed by its use. In teams of 2-4, undergraduate students tackled the case and provided novel research ideas, which may hold the key to many unanswered questions regarding the positive and negative aspects of cannabis use. In total, the 2018-2019 USCC attracted 861 undergraduate students from 14 universities across Canada and the United States. The top $25 \%$ of written submissions from each participating campus are highlighted in this abstract booklet.
\end{abstract}

Keywords: cannabis; cannabidiol; tetrahydrocannabinol; undergraduate research; science case competition

Table of Contents

Top 25\% Scinapse USCC Abstracts.

pg. A01-A18

\section{Conference Abstracts}

Note: These abstracts have been reproduced directly from the material supplied by the authors, without editorial alteration by the staff of the URNCST Journal. Insufficiencies of preparation, grammar, spelling, style, syntax, and usage are the authors.

\section{Top $25 \%$ Scinapse USCC Abstracts}

The effect of cannabidiol administration to heroin-addicted rats in the withdrawal phase on muscle cramping Erin Artna [1], Amy Huang [1], Aabroo Kaur [1], Hannah Kim [1], [1] McMaster University, Hamilton, Ontario, Canada L8S 4L8

The medicinal properties of cannabis are a pressing topic in modern research, notably those of one particular ingredientcannabidiol (CBD). Due to CBD's antipsychotic property and minimal abuse potential, there is prospective viability in CBD as a treatment option for addiction-associated symptoms. One of the most debilitating symptoms associated with withdrawal is severe muscle cramps. This research proposal aims to explore the following hypothesis: the administration of CBD to heroin addicts in the withdrawal phase will alleviate the intensity of muscle cramps they exhibit. CBD works to inhibit adenylyl cyclase (AC)/cAMP-associated signalling which counteracts the increased AC production caused by heroin tolerance. Resultantly, noradrenaline levels are restored to normal production and the symptom of muscle cramps is reduced. The testing of the hypothesis will require the use of animal models. Norway Rats will be injected heroin for seven days to incubate symptoms of addiction before stopping dosage before being administered CBD to test its success in reducing withdrawalinduced muscle cramps. 
UNDERGRADUATE RESEARCH IN NATURAL AND CLINICAL SCIENCE AND TECHNOLOGY (URNCST) JOURNAL Read more URNCST Journal articles and submit your own today at: https://www.urncst.com

Epigenetic impacts of cannabis on varying age groups of schizophrenic individuals Maleka El Naghi [1], Liberty Liu [1], Pooja Manglorkar [1], Isha Verma [1]

[1] McMaster University, Hamilton, Ontario, Canada L8S 4L8

Cannabidiol (CBD) is currently informally used as an adjunctive treatment for schizophrenia due to its role in enhancing the effect of endocannabinoids, relieving schizophrenic symptoms. As therapeutic potential of CBD has been popularized recently, there has not been sufficient research on the risks of using CBD therapy. We propose research on the negative epigenetic effects of using CBD therapy for schizophrenia, and which age groups are most susceptible to change. The epigenetic change that occurs is related to an increase in transcription of Fatty-acid amide hydrolase (FAAH). The protein responsible for enzymatic degradation of endocannabinoids in attempt to balance the response to normal levels after the increased efficacy of endocannabinoids due to CBD. This will decrease endogenous cannabinoid levels, ultimately increasing schizophrenic symptoms. Especially as Canada has recently legalized cannabis for recreational use, the general public has easier access to CBD for therapy. We wish to study the epigenetic effects of using CBD as a treatment in different age groups of schizophrenic mice to determine which age groups are at highest risk in using CBD treatment.

Facilitating the bloom of intestinal flora: Observing the influence of tetrahydrocannabinol (THC) on beneficial bacteria inhabiting the intestinal microbiota Simone Gindin [1], Esha Karia [1], Alisiya Petrushkevich [1], Shadi Sadeghian [1] [1] McMaster University, Hamilton, Ontario, Canada L8S 4L8

Healthy human bodies are inhabited by over 100 trillion bacterial cells. The study of the symbiotic relationship between humans and these unicellular organisms has become of great importance in the medical field. In fact, the pathology of many diseases involves perturbation of the intestinal flora. Tetrahydrocannabinol (THC), the psychotropic ingredient of cannabis, has caught the attention of healthcare researchers given its ability to increase appetite, and reduce nausea and inflammation. THC has also been associated with modulating the effects arising in the endocannabinoid system, however, its ability to affect bacteria growth has not been studied. This proposed experiment seeks to explore the growth and vitality of beneficial gut microbiota (Lactobacillus acidophilus, Akkermansia muciniphila, Bifidobacterium bifidum, and Streptococcus thermophilus) upon exposure to THC. Through utilizing an experimental approach, beneficial bacterial cultures will be collected to study the growth of the microorganisms incubated with THC, relative to a positive and negative control. The results of the proposed study could inform future experiments investigating the treatment of a range of diseases with cannabis. The benefits of these treatments could also be extended to improving the daily health of the general population.

Biodegradable hemp plastic as a model for bioplastics, sourced from marijuana byproduct Maisha Ahmed [1], Christy Au-Yeung [1], Bushra Haque [1], Peiyao Wang [1] [1] McMaster University, Hamilton, Ontario, Canada L8S 4L8

Over time, society has evolved to become dependent on the production of plastics for various applications despite detrimental environmental and health impacts. However, the production of bioplastics using Cannabis sativa (hemp) has shed light on an alternative, renewable resource that decreases fossil fuels, oils and natural gas emissions. In October 2018, Canada legalized the use of Cannabis indica (marijuana), which is closely related to hemp. This introduces prospects of newly derived forms of bioplastics, specifically through the use of marijuana by products. The inevitable integration of marijuana into society makes it an indisputable resource for sustainability in Canada.

Treatment of THC overconsumption using a novel compound Saud Haseeb [1], Winston Hou [1], Matthew Lynn [1], Abirami Sudharshan [1] [1] McMaster University, Hamilton, Ontario, Canada L8S 4L8

The legalization of cannabis in Canada has triggered increased concern for the potential adverse effects of its consumption. Tetrahydrocannabinol, the primary psychoactive component of cannabis, is an agonist for the cannabinoid type receptor, and has been shown to inflict acute toxicity on the central nervous system when ingested in high concentrations. The demand for treatments countering these toxic effects is actively rising. A promising prospective solution can be found in compounds that act as inverse agonists to the CB1 receptor, inhibiting its constitutive activity. One such compound, 6-benzhydryl-4-aminoquinolin-2-ones, exhibits prolific inverse agonism. We propose the use of mice in an in vivo model to evaluate the abilities of

Cyr et al. | URNCST Journal (2019): Volume 3, Issue 9

DOI Link: https://doi.org/10.26685/urnest.163

Page A2 of A19 
UNDERGRADUATE RESEARCH IN NATURAL AND CLINICAL SCIENCE AND TECHNOLOGY (URNCST) JOURNAL Read more URNCST Journal articles and submit your own today at: https://www.urncst.com

6- benzhydryl-4-amino-quinolin-2-ones as an inverse agonist in mitigating the adverse effects of cannabis. This study would pave the way for future research in developing a pharmacological treatment for the acute toxic effects of excessive cannabis consumption.

\author{
Modelling optimal light intensity, spectra and photoperiod length for optimal Cannabis indica growth and THC, CBD \\ production \\ Alex Chen [1], Kevin Du [1], Gina So [1], Denesh Peramakumar [1] \\ [1] McMaster University, Hamilton, Ontario, Canada L8S 4L8
}

Cannabis indica is one of two species of cannabis, which produces tetrahydrocannabinol (THC) and cannabidiol (CBD) used for medicinal and recreational purposes. $C$. indica has also been identified as clinically useful for a variety of purposes. $C$. indica also has a high ratio of cannabidiol (CBD) to THC. The CBD acts as an antagonist on cannabinoid receptors mitigating the psychoactive effects of THC, which acts as a partial agonist. Despite its favorable effects, there is little known about the ideal growth conditions of $C$. indica in indoor environments, which are commonly used by producers to control growing conditions. Lighting is one of the key variables involved with the growth of cannabis; it affects the yield, cost and environmental impact of cannabis growth. Therefore, there is value in modelling the impacts of different lighting environments on the development of $C$. indica. The goal of this study is to analyze the effect of differing light spectra, day/night cycles and light intensities on cannabis growth as well as the changes in THC and CBD yields.

Using knockout mice models to explore links between THC and schizophrenia Sayed Azher [1], Keerat Grewal [1], Shaun Jim [1], Harmy Thakar [1]

[1] McMaster University, Hamilton, Ontario, Canada L8S 4L8

The legalization of marijuana raises many concerns regarding its consequences on the central nervous system. From being widely used as a medicinal remedy to now being used recreationally, this proposal aims to focus on an experiment which explores THC and its potentially detrimental effects on the brain, specifically in mice at-risk for schizophrenia. This proposal outlines a mouse model analysis of an analogue of THC that will be constructed using a knockout mice lineage in which the mice have a genetic predisposition to schizophrenia and lack the CB1 receptors responsible for binding THC. The resultant mice will undergo a grooming behaviour test and an elevated zero maze test which will then be compared with other experimental and control groups. This will allow for the direct observation of THC's effects on the brain, providing insight on the consequences of the legalization of marijuana and its recreational use.

\title{
An alternative treatment to ADHD: The role of cannabidiol as a 5HT1A receptor agonist Hafsa Idrees [1], Shomaila Rashid [1] [1] McMaster University, Hamilton, Ontario, Canada L8S 4L8
}

Impulsivity associated disorders such as attention deficit hyperactivity disorder and borderline personality disorder are known to result from irregular modulation of dopamine systems in the prefrontal cortex region of the brain. Although amphetamines such as Adderall are regularly used as treatment options for such mental illnesses, they have a number of undesirable side effects and produce inconsistent effects. As an alternative, this application proposes to investigate the use of cannabidiol, a non-psychoactive component of cannabis as a treatment. The usage of cannabidiol as a treatment option relies on the role of 5HT1A receptors in the brain play in modulating dopamine, and on cannabidiol's own role as a 5HT1A activator. We propose to investigate the effect of cannabidiol in impulsivity prone rats through observations from a delayed reinforcement task and from functional magnetic resonance imaging (fMRI) to detect activity changes in the brain.

Proposal on the relationship between using cannabis, consuming isothiocyanate rich broccoli and HDAC expression in schizophrenia Saihajleen Dhillon [1], Natasha Verhoeff [1]

[1] McMaster University, Hamilton, Ontario, Canada L8S 4L8

Schizophrenia is a chronic brain disorder which affects the thoughts, feelings, and behaviour of people. According to current studies, there is a correlation between schizophrenia and amounts of the epigenetic marking histone acetylation. Schizophrenic 
UNDERGRADUATE RESEARCH IN NATURAL AND CLINICAL SCIENCE AND TECHNOLOGY (URNCST) JOURNAL Read more URNCST Journal articles and submit your own today at: https://www.urncst.com

patients show high HDAC levels which leads to reduced amounts of acetyl markings and subsequent tightening of the chromatin structure. Additionally, THC, a psychoactive compound present in cannabis, has been found to also cause increased HDAC activity, and several studies have shown a relationship between using cannabis and the development or worsening of schizophrenia, however, the exact molecular mechanisms are unknown. Isothiocyanates, a class of organic compounds found in herbs like broccoli, have been found to be HDAC inhibitors. This study will test the relationship between cannabis, isothiocyanates, and schizophrenia at the genetic level by using normal and DISC1 mice, and ChIP, Sequencing, RT-(q) PCR, and Western Blot. It is hypothesized that if schizophrenic patients eat broccoli sprouts, their HDAC will be inhibited, genes responsible for cognitive function will be euchromatin, and schizophrenic people will have improved cognition and functional outcomes. It is also hypothesized that if people use cannabis, their HDAC will be more expressed, genes responsible for cognitive function will be heterochromatin, and there may be a higher chance of the development or worsening of schizophrenia. Depending on which genes are affected and how much they are affected, understanding an epigenetic mechanism between cannabis and schizophrenia may help determine why it can be harmful, and how molecules like isothiocyanates can potentially reduce the harm.

The use of cannabis-derived essential oils as a novel acaricide targeting Varroa destructor-induced Apis mellifera colony collapse disorder

Megan Cheng [1], Alison Hacker [1], Rhea Jangra [1]

[1] McMaster University, Hamilton, Ontario, Canada LSS $4 L 8$

As the production of marijuana and marijuana-based products becomes legalized, several novel applications of Cannabis sativa have begun to emerge. In particular, growing evidence has suggested that essential oils (EOs), derived from different cultivars of industrial hemp, might be capable of managing insect pest infestations in organic crops. Likewise, there is accumulating evidence that these EOs are capable of targeting pests without harming non-target invertebrates. Therefore, we propose the development and use of a novel EO, derived from the industrial hemp cultivar, Felina 32, in the treatment of Varroa mite (Varroa destructor) infestations within honey bee (Apis mellifera) colonies. We will extract EOs from hemp inflorescences utilizing steam distillation. Gas chromatography-mass spectrometry will be used to analyze the composition of this hemp oil. Aerosol application of varying concentrations of our EO product will be used to treat Varroa mite-infested Apis mellifera colonies. From these data, toxicity and effective dosage of this novel acaricide will be determined. This project will serve to address pesticide resistance and Varroa mite-induced Apis mellifera colony collapse, both of which are growing concerns within the agricultural industry worldwide.

Cannabinoids and labour pain relief

Colleen Dawson [1], Ashlee Gonsalves [1], Sofia Ivanisevic [1], Sarah Yang [1]

[1] McMaster University, Hamilton, Ontario, Canada L8S 4L8

Cannabinoids have gained large interest in the research field for potential therapeutic implications against pain. It has been proved to be beneficial for treatment of chronic pain, however, little is known about treatment for more hyper acute pain, in particular, as an alternative for the use of epidural analgesia during labour. Although epidurals are very effective and commonly used, they bring accessibility issues and side-effects. Thus, it proposes an investigation into the use of cannabinoids, specifically cannabidiol (CBD), to mediate labour pain. We propose a study using rat models, injected with either CBD oil, epidural analgesia, or a saline control during labour. Rodent pain levels will be evaluated with the Grimace Scale. Average scores between control and experimental groups will be analyzed to evaluate the effectiveness of CBD oil for pain relief, as well as to compare its effects to epidural analgesia. There are, however, some limits to this study, such as its inability to test for long term effects of using cannabinoids during labour on infants postnatally.

Solid lipid nanoparticles: A novel cannabinoid delivery mechanism for multiple sclerosis treatment Milena Cioana [1], Dorsa Kord [1], Lucy Lan [1], Michael Shi [1]

[1] McMaster University, Hamilton, Ontario, Canada L8S 4L8

Multiple sclerosis (MS) is an autoimmune disorder affecting the central nervous system, damaging the myelin sheath and axons, impairing neurotransmission. Previous studies have shown cannabis and synthetically derived cannabinoids, such as HU-211, as effective treatments for pain and spasticity in MS. However, due to limitations in drug chemistry and delivery, the long-term effects of HU-211 on MS have not been evaluated. Solid lipid nanoparticles (SLN) demonstrate characteristics such as

Cyr et al. | URNCST Journal (2019): Volume 3, Issue 9

DOI Link: https://doi.org/10.26685/urncst.163

Page A4 of A19 
UNDERGRADUATE RESEARCH IN NATURAL AND CLINICAL SCIENCE AND TECHNOLOGY (URNCST) JOURNAL Read more URNCST Journal articles and submit your own today at: https://www.urncst.com

improved bioavailability, prolonged drug release has great potential to carry HU-211 to the CNS. Our study is the first study that aims to employ a solid lipid nanoparticle mechanism to effectively deliver HU-211 to the central nervous system as a treatment for multiple sclerosis. We propose to use EAE mouse models to determine the effectiveness of HU-211 delivered through SLN on MS disease progression.

Investigating increased potential stroke or heart disease risk among cannabis and oral contraceptive users Ryan Au [1], Sam Liu [1], Enoch Tin [1], Jonas Yeung [1]

[1] McMaster University, Hamilton, Ontario, Canada LSS 4L8

As a result of the legalization of marijuana (cannabis) in Canada, we expect a higher prevalence of simultaneous use of cannabis and oral contraceptives (OC). Concurrent use of both substances may exhibit physiological consequences. Our study will examine the potential synergistic effects of cannabis and OC on blood coagulation, which increases the risk of ischemiamediated diseases such as stroke. To the best of our knowledge, there have been no studies on the simultaneous use of OC and cannabis. Current scientific evidence suggests the individual consumption of either drug increases the risk of blood coagulation. $\mathrm{OC}$ is known to elevate fibrinogen concentrations, whereas cannabis stimulates platelet glycoprotein IIb/IIIa expression. The upregulation of these components promotes platelet aggregation or blood clotting. The predicted interaction between each individual drug is hypothesized to cause synergistic effects for blood coagulation. A mouse study will be conducted to investigate the mechanism of action and synergistic effects. Mice samples will be administered with either no drug, OC, cannabis, or both. ELISA and flow cytometry will be used to quantify fibrinogen and platelet glycoprotein IIb/IIIa, respectively. This will ensure that the expression of these components is consistent with individual pathways. Confocal microscopy techniques will be used to quantify the amount of platelet aggregation through the co-localized fluorescent signal. The possibility of synergistic effects by OC and cannabis will be assessed with fluorescence.

Utilizing fibroblast-derived hepatocyte-like cells to implement a double-humanized mouse model to represent human 49-THC metabolism in a mouse

Tyrah Ritchie [1], Daniel Shields [1], Anika Spasov [1]

[1] McMaster University, Hamilton, Ontario, Canada L8S 4L8

Ascertaining marijuana's short and long-term effects has become critically important with the recent legalization of cannabis. Key metabolic differences existing between mice and humans generate uncertainty as to whether findings discovered through murine models can be extrapolated to humans. This is particularly relevant in marijuana research, which often adopts murine models, since the main metabolizers of tetrahydrocannabinol ( $\Delta 9$-THC) differ between species. Most $\Delta 9$-THC metabolism occurs in the liver through the activity of enzymatic hemeproteins, known as cytochrome P450s (CYPs). However, the CYP families differ between species: in humans CYP2C9, CYP2C19 and CYP3A4 metabolize $\Delta$ 9-THC, while in mice CYP2C29 and CYP3A11 are responsible. Consequently, the main marijuana metabolites and their respective concentrations vary between species, which may cause disparate effects in mice and humans. Thus, there is need to improve system validation to ensure results are comparable. A relatively new murine model that has garnered much attention in scientific research is the doublehumanized mouse model (DHMM) which contains a fully functioning human liver and immune system in Fah-/-, RAG2-/-, and IL2R $\gamma$-/- mice. However, this method utilizes primary human hepatocytes (PHHs), which are often expensive and inaccessible. The limited feasibility of this model may explain why it has not yet been applied to marijuana research. Thus, it is proposed to utilize fibroblast-derived hepatocyte-like cells, which are analogous to PHHs, to produce the DHMM. This will improve accessibility to the humanized model and form an applicable model to human metabolism, thus advancing the validity of marijuana research as an entire field.

Development of a novel paper-based enzyme-linked immunosorbent assay for roadside detection of THC and THCCOOH in oral fluid

Jessica Chee [1], Judy Chen [1], Harry Li [1], Jim Xie [1]

[1] McMaster University, Hamilton, Ontario, Canada L8S 4L8

The legalization of cannabis in Canada has created numerous concerns regarding its regulation. In particular, drugged driving presents a major public health safety concern. Although legal limits have been established for cannabis intake in drivers, the lack of precise and accurate roadside detection methods for cannabis hinders lawful enforcement of these regulations. The current diagnostic procedure for roadside detection of cannabis begins with either a standardized field sobriety test or an oral

Cyr et al. | URNCST Journal (2019): Volume 3, Issue 9

Page A5 of A19

DOI Link: https://doi.org/10.26685/urnest.163 
UNDERGRADUATE RESEARCH IN NATURAL AND CLINICAL SCIENCE AND TECHNOLOGY (URNCST) JOURNAL Read more URNCST Journal articles and submit your own today at: https://www.urncst.com

fluid (OF) test for $\Delta 9$-tetrahydrocannabinol (THC). Drivers who test positive for either of these assessments may subsequently be subjected to a THC blood test. These tests are all limited in their accuracy or ease of access. This proposal aims to develop a novel detection method for blood THC that is rapid, feasible, and sensitive. OF levels of THC and one of its metabolites, 11nor-9-carboxy-THC (THC-COOH), will be correlated with legal limits of blood THC to determine OF cannabinoid thresholds. The amount of antibodies required to detect these thresholds will then be determined using enzyme-linked immunosorbent assays (ELISAs) and be used to develop a paper-based immunoassay. The proposed method will address the challenges of current practice, including low sensitivity and OF contamination by smoking, and be able to accurately predict blood THC levels on the roadside.

Investigation into cannabidiol's modulation of withdrawal symptoms in opioid-dependence Joeffre Braga [1], Angad Hanjrah [1], Damien Joseph [1] [1] McMaster University, Hamilton, Ontario, Canada L8S 4L8

Over the past 20 years an opioid epidemic has been observed. Pharmaceutical advancements are required to reduce the misuse of opioids and the prevalence of addiction. Cannabinoids have been studied for their opioid-sparing effects and ability to inhibit reward- facilitating effects. However, research surrounding their capacity in easing withdrawal symptoms is lacking. Certain cannabinoids have been implicated in the reduction of withdrawal behaviour in animal models, but a particular mechanism is yet to be identified. Various studies suggest that the hyperactivity of noradrenergic locus coeruleus (LC) neurons significantly contributes to withdrawal symptoms. Additionally, it has been reported that this hyperactivity is largely mediated by an excitatory amino acid (EAA) input to the LC and that 5-HT selectively attenuates excitation of LC neurons by EAAs. We believe that CBD, typically the second most abundant cannabinoid in the Cannabis sativa plant, has potential as a novel treatment for withdrawal. This claim is founded on two main findings: i) CBD enhances 5-HT neurotransmission and ii) CBD does not induce conditioned place preference. Therefore, we hypothesize that CBD has the capacity to reduce the EAA-induced excitation of the LC neurons and avoid causing its own withdrawal symptoms. Here, we propose an experimental workflow using morphine-dependent animal models in two phases to investigate this phenomenon. The first phase will use electrophysiological recordings of single LC neurons to observe modulation of excitation when CBD is administered during opiate withdrawal. The second phase will assess changes in withdrawal behaviours when CBD is administered.

Development of a novel aptamer designed to reduce THC induced psychoactivity in the brain Angela Dong [1], Adriana Fedorowycz [1], Ramy Gabarin [1], Kevin Shao [1]

[1] McMaster University, Hamilton, Ontario, Canada L8S 4L8

To our knowledge, there is no drug available that attenuates the psychoactivity of marijuana in active users. Some users report instances of panic attacks, severe paranoia, and even temporary symptoms of psychosis as side effects that manifest following drug administration, and would benefit from a means to quickly reduce the active concentration of psychoactive compounds tetrahydrocannabinol (THC) and its primary active metabolite 11-hydroxy- $\Delta 9$ - tetrahydrocannabinol (11-OH-THC) in the brain. Aptamers - oligonucleotide sequences capable of binding to small molecules with high affinity and specificity present a promising solution. Evidence suggests that aptamers administered intravenously can cross the blood-brain barrier (BBB) in order to target THC and 11-OH-THC concentrations in the brain. Aptamer binding will inhibit the interaction of the aforementioned psychoactive compounds with their target receptor, cannabinoid receptor 1 (CB1), effectively reducing psychoactivity. Using the SELEX process, we will generate two aptamers designed for binding to THC and 11-OH-THC, respectively. In vitro binding assays will be used to evaluate the aptamers' affinity and specificity. Finally, an in vivo study with accompanying ex vivo analysis will assess the distribution of the aptamers in mice following intravenous administration, as well as their ability to bind to their target molecules.

Determining a novel mechanism to elucidate levels of inebriation by cannabis Michael Chan [1], Joseph D'Ercole [1], Alexander Mastrolonardo [1], Yuanxin Xue [1] [1] McMaster University, Hamilton, Ontario, Canada L8S 4L8

In light of the recent legalization of cannabis in Canada, there is an increasing need to better understand its effects and establish appropriate guidelines surrounding its use. Particularly, driving under the influence of cannabis is a leading cause of roadrelated injuries and deaths in Canada. Current drug tests utilized by law enforcements have assisted in quantifying the degree of inebriation, however the tests have significant flaws that would undermine their reliability and validity. The following study

Cyr et al. | URNCST Journal (2019): Volume 3, Issue 9

DOI Link: https://doi.org/10.26685/urnest.163 


\section{UNDERGRADUATE RESEARCH IN NATURAL AND CLINICAL SCIENCE AND TECHNOLOGY (URNCST) JOURNAL}

Read more URNCST Journal articles and submit your own today at: https://www.urncst.com

hopes to correlate levels of inebriation to those of a chemical tracer found in both saliva and the brain. To accomplish this, a rat model must be developed, in which a range of functional tests will be utilized, along with fMRI, to determine the level of functional deficits experienced in rats following exposure to varying degrees of cannabis smoke. Afterwards, rats will be euthanized, and their neural, salival, and blood samples will be taken. These samples will be analyzed using gas chromatography and mass spectrometry to quantify the presence of THC and its metabolites. Having determined the relevant concentrations within various rat tissues, statistic models will be applied to evaluate potential relationships between concentrations and performance on functional tests or fMRI results. This may lend insight into effective methods and tests capable of reliably predicting significant psychological changes by analyzing THC and metabolite concentrations. This research will pave the way in determining new guidelines for the detection of cannabis-related compounds in individuals suspected of driving under the influence.

\section{Development of a novel THC detection method Elger Baraku [1], Klara Stringa [1], Sissi Yang [1] [1] McMaster University, Hamilton, Ontario, Canada LSS $4 L 8$}

Cannabis contains the psychoactive cannabinoid $\Delta 9$-tetrahydrocannabinol (THC). Such psychoactivity has been shown to reduce psychomotor performance leading to impaired driving and its resulting dangers and consequences. To regulate cannabis consumption and eliminate impaired driving, detection methods have been developed with limited success due to either low sensitivity or low specificity. To develop a more reliable detection method, we propose to use an immunoassay with anti-THC antibodies to pre-concentrate THC while increasing the sensitivity of our method. Further, a screen-printed electrode would be used to detect the THC from the pre-concentrated sample, increasing the specificity of our method. Stock solutions with known THC concentrations and solutions with common interferences found in saliva would be tested for specificity. False positives and false negatives would be compared to true positives and true negatives to validate the selectivity and specificity of our THC detection method as well as determine its limit of detection (LOD). It is expected that our method would have high selectivity, sensitivity, accuracy, and a low LOD due to its hybrid analytical nature. In determining the significance of this method in detecting THC at low concentrations with possible interferences, clinical trials can explore its success at detection with human saliva samples. Ameliorating detection methods of THC could aid in the development of detection methods for other compounds. Ultimately, this can reduce incidences of impaired driving and can help in the safe transition of road regulations and policies with the legalization of cannabis.

\section{THC and alpha-pinene}

Mila Huhtala [1], Alec Mazurek [1], Matthew Shammas-Toma [1]

[1] Western University, London, Ontario, Canada N6A $3 K 7$

The current study aimed to investigate tetrahydrocannabinol (THC) and $\alpha$-pinene; two components of the cannabis plant. Specifically, we are interested in the possible synergistic effects of these two compounds on the respiratory system. THC has been shown to have bronchodilatory effects when administered through a nebulizer, and previous research has shown its results comparable to Salbutamol (Ventolin), a common treatment in reactive airway diseases such as asthma and chronic-obstructive pulmonary disorder (COPD). $\alpha$-pinene is a natural derivative of the cannabis plant, and in its pure form is also known to have bronchodilatory effects. The purpose of this study was to investigate whether the synergy of $\alpha$-pinene and THC would produce greater overall bronchodilatory effect than when compared to pure THC or Salbutamol. It would be predicted that since both pure forms exhibit significant bronchodilation effects, their combined mechanism would be more powerful than either on its own. The compounds will be introduced to 30 mice, with 10 being administered pure THC aerosol, 10 the salbutamol aerosol, and 10 the THC/ $\alpha$-pinene combination aerosol. Forced Oscillation Technique (FOT) will be employed to analyze the results of each administration. The hypothesis will be supported if the FOT measure from the THC/ $\alpha$-pinene group confirms an average larger bronchodilation in its subjects than either of the pure THC or Salbutamol groups. 
UNDERGRADUATE RESEARCH IN NATURAL AND CLINICAL SCIENCE AND TECHNOLOGY (URNCST) JOURNAL Read more URNCST Journal articles and submit your own today at: https://www.urncst.com

The inhaled delivery of AM-6545 as a novel method of reducing the risk of chronic bronchitis in mice exposed to THC through cannabis smoke: A research proposal

Tirth Patel [1], Keval Raval [1], Jay Shah [1], Tania Wirring [1]

[1] Western University, London, Ontario, Canada N6A $3 K 7$

The recent legalization of cannabis in Canada has increased concern over its impact on the respiratory system. Tetrahydrocannabinol (THC) binds to cannabinoid receptors within the endocannabinoid system. The binding of THC to cannabinoid receptors in the lungs causes the inflammation of bronchial tubes, leading to chronic bronchitis. Pulmonary fibrosis and chronic bronchitis are a result of the inflammation of the connective tissue within alveoli and bronchial tubes respectively. Current research advancements used in pulmonary fibrosis treatments in mice will allow the use of AM-6545 to reduce the inflammation of bronchial tubes. This proposal suggests that the use of an inhaled delivery system for THC exposure and the administration of AM-6545 in mice will provide a novel approach to reduce bronchoconstriction. As a cannabinoid receptor antagonist, AM-6545 inhibits the binding of THC to cannabinoid receptor type 1 (CB1) in the lungs. In addition, AM-6545 does not affect neurotransmission in the central nervous system (CNS), thus, having no effect on the dopamine over-release that characterizes the high. The proposed experiment is to test the effectiveness of AM-6545 using mice. By comparing a control group that is exposed to THC, with an intervention group that is exposed to both THC and AM-6545. The differences in bronchial tube inflammation can be measured. A reduction in bronchial tube inflammation in the intervention group would indicate that AM-6545 reduces the risk of chronic bronchitis due to bronchoconstriction in mice.

HU-308: A novel drug therapy targeting cannabinoid receptors for combating atherosclerotic cardiovascular disease Christine Cha [1], Sherry Cui [1], Ushra Khan [1], Justin Kim [1]

[1] Western University, London, Ontario, Canada N6A $3 K 7$

Atherosclerotic cardiovascular disease (ASCVD) is a chronic inflammatory condition that involves the upregulation of the immune system response to arterial low-density lipoprotein (LDL) accumulation. Atherosclerotic plaque formation begins with the transendothelial migration (TEM) of monocytes into the arterial intima. Following maturation of the monocytes into macrophages, the macrophages phagocytose the accumulated LDL and consequently transform into foam cells. The amalgamation of foam cells and LDL deposits then form the early atherosclerotic plaque. Recent studies have shown that the endocannabinoid system can modulate the progression of atherosclerosis. Activation of cannabinoid receptor two (CB2) appears to play a protective role by impeding TEM and reducing inflammation. Moreover, the selective upregulation of CB2 in atherosclerotic plaques compared to healthy tissue renders it a promising target for drug therapy. In this proposal, we plan to use various concentrations of HU-308, a potent selective CB2 agonist, on LDLR -/- mice to evaluate its efficacy in hindering plaque formation. We plan to utilize ultrasound and immune histological techniques to measure ASCVD progression in response to the varying HU-308 dosages.

Apoptotic effects of cannabidiol on benign meningioma

Renee Kongv [1], Elaine Liu [1], Ananya Manvik Aneja [1], Elizabeth Wei [1]

[1] Western University, London, Ontario, Canada N6A $3 K 7$

Meningiomas, common benign tumors that form from membranes surrounding the brain and spinal cord, are particularly harmful to patients due to its location. These tumours can put pressure on vital areas of the brain leading to life threatening neurological problems. Currently, surgery and radiotherapy exist for such tumours; however, these treatments are invasive or have a high associated cost. They can also lead to long-term neurological problems in patients. Alternatively, cannabis has been shown to be effective in reducing the growth of cancerous tumours. It has been reported that the active components of cannabis, namely cannabidiol (CBD), can inhibit cell growth. Thus, it is hypothesized that the apoptotic effects of CBD can potentially be beneficial in treating meningiomas. Therefore, this experiment aims to compare the levels of apoptosis in meningioma cell lines treated with cannabidiol and the traditional radiation treatment, using TUNEL assay. Even if further in vivo studies on CBD's effect is required for a conclusive application as a novel treatment of meningiomas, the potential for an alternative, more cost-effective, and less invasive treatment is promising. If cannabidiol is successful in inducing a significant apoptotic effect, the use of CBD could become a novel treatment of benign human meningiomas.

Cyr et al. | URNCST Journal (2019): Volume 3, Issue 9

Page A8 of A19

DOI Link: https://doi.org/10.26685/urnest.163 
UNDERGRADUATE RESEARCH IN NATURAL AND CLINICAL SCIENCE AND TECHNOLOGY (URNCST) JOURNAL Read more URNCST Journal articles and submit your own today at: https://www.urncst.com

Feasibility of Chlamydomonas reinhardtii in the heterologous biosynthesis of $\Delta 9$ - tetrahydrocannabinol Ira Brown [1], Jonah Rakoff [1]

[1] Western University, London, Ontario, Canada N6A $3 K 7$

Prevailing liberal trends in favour of the legal use of cannabis have led to an increase in demand for the plant and its psychoactive compound THC. Current agricultural methods of scaling up the production of THC are limited by the physical space and time required to grow cannabis. Additionally, environmental issues and financial inefficiency remain lingering concerns. This proposed study focuses on a possible alternative method of THC production, specifically the biosynthesis of THC using genetically modified Chlamydomonas reinhardtii. The glass bead method will be used to transform $C$. reinhardtii using three distinct vectors, each carrying an enzyme involved in the biosynthetic pathway of THC. Following incubation of cells in a medium containing starting material for the biosynthesis, THCA, the immediate precursor to THC, will be isolated and quantified. Although the demand for whole dried cannabis would likely remain high, biosynthetically produced THC would provide a cost-effective and environmentally-friendly option for the preparation of THC pharmaceuticals and recreational products. If this method proves feasible, the process of using genetically-modified algae to produce THC can be modified to produce other cannabinoids.

Moderation of the psychoactive effects of $\Delta$ 9-tetrahydrocannabinol using transcutaneous administration of cannabidiol Meet Panchal [1], Ansh Patel [1], Samaunus Safa [1]

[1] Western University, London, Ontario, Canada N6A $3 K 7$

$\Delta 9$-Tetrahydrocannabinol (THC), the main psychoactive ingredient in cannabis, has been discovered to cause cognitive defects and long-term behavioral alterations. These include recognition memory deficits, social withdrawal, altered emotional processing and problems with working memory. Long-term exposure to THC also affects higher order executive function tasks, such as the ability to plan, organize, solve problems, make decisions. This becomes a more prevalent issue considering that the negative effects of THC are most severe in adolescents because regions of their brain involved with executive function and learning are still undergoing development. Furthermore, studies conducted by ElSohly et al. (2016) found that the average ratio of THC to cannabidiol (CBD) in marijuana strains has increased from 14 times in 1995 to 80 times in 2014. Studies have shown that CBD can counteract the negative effects of THC on both behavioral and neurochemical levels even if it is applied before or after the THC has been inhaled. This proposal suggests that the transcutaneous application of CBD, via a patch, may allow users to experience similar psychoactive effects, but it will mediate the duration of the effects and may reduce the risk of longterm cognitive defects. Transcutaneous absorption is a slower method of delivery relative to inhalation, meaning individuals can still enjoy the recreational use of marijuana with a decreased risk of long-term cognitive and behavioral alterations.

\section{Reducing cadmium uptake in Cannabis sativa with Agrobacterium tumefaciens-mediated ATPase transformation Richard Cheng [1], Yixin Jiang [1], Thomas Milazzo [1], Patrick Yao [1] \\ [1] Western University, London, Ontario, Canada N6A $3 K 7$}

The recent legalization of Cannabis sativa in Canada has introduced concerns regarding its effect on human health. C. sativa is identified as a good bioaccumulator of heavy metals. Unfortunately, this property may compromise its safety as a commercial product because the bioavailability, carcinogenicity, and volatility of heavy metals increases when smoked. Therefore, the capacity of the plant to accumulate heavy metals such as cadmium may present pathological concerns for users. We propose the introduction of two novel proteins using Agrobacterium-mediated transformation to reduce the plant's bioaccumulative capacity as the intrinsic mechanisms of cadmium ion uptake and processing remain relatively unstudied in $C$. sativa. AtABCC1 is a critical protein for vacuole transport and subsequent sequestration of cadmium, and ZntA is a cadmium efflux pump of bacterial origin. These proteins may decrease concentrations of cadmium in the leaves and the buds of the plant, effectively reducing the pathogenicity of $C$. sativa. Future work should identify the function of genes involved in the mechanism of bioaccumulation. 
UNDERGRADUATE RESEARCH IN NATURAL AND CLINICAL SCIENCE AND TECHNOLOGY (URNCST) JOURNAL Read more URNCST Journal articles and submit your own today at: https://www.urncst.com

Reduction of cadmium and lead uptake in Cannabis sativa through application of nano-hydroxyapatite in soil Isaac Alexander-Cook [1], Joshua Garcia-Barrios [1], Brandon McMurray [1], Caleb Shaver [1]

[1] University of Waterloo, Waterloo, Ontatio, Canada N2L 3G1

The advent of legalized cannabis production in Canada has heightened concerns around contaminant presence in commercially grown and homegrown crops. Heavy metals are contaminants of key concern due to the significant potential for heavy metal accumulation in cannabis plants. This study aims to observe the effectiveness of reducing the uptake of cadmium and lead in Cannabis sativa plants by use of nano-hydroxyapatite (nHAp). This study hypothesizes that an increased soil concentration of nHAp will promote the immobilization of cadmium and lead in soil mediums reducing their uptake in $C$. sativa. Lower concentrations of cadmium and lead are expected to be found in plant matter of $C$. sativa grown in nHAp-treated soil. This method of applying nHAp to $C$. sativa could provide commercial producers and home growers with a method for reducing toxic contaminants in products.

Action of cannabidiol (CBD) and $\Delta 9$-tetrahydrocannabinol ( $\triangle 9$-THC) in treatment for colorectal cancer Alyssa Huang [1], Jeremy Wang [1], Luong Vu Son [1]

[1] University of Waterloo, Waterloo, Ontatio, Canada N2L 3G1

Colorectal cancers are currently the third most common type of cancer in the world and second most common type of cancer in Canada, with a 5-year net survival rate of 64\%. Furthermore, the World Health Organization has stated that over 13 million deaths due to cancer is projected to be reached in 2030. Given these numbers, there is understandably significant interest in developing novel adjuvant treatments for these common cancers. Of particular interest are compounds present in Cannabis sativa L.: cannabinoids and their derivatives cannabidiol (CBD) and the psychotropic agent $\Delta 9$-tetrahydrocannabinol $(\Delta 9$ THC). There is a substantial body of research identifying the role of cannabinoids in inducing apoptosis and autophagy in tumour cells, but relatively little of it focuses on colorectal cancer cells. Herein we will be examining the role and pathway by which cannabinoid-induced apoptosis occurs in colorectal cancer cells. We hypothesize that interaction between TRPV2 channels and CBD and $\triangle 9$-THC cannabinoids trigger apoptosis in human colorectal cancer cells.

Effects of the inhalation of fenvalerate combustion products on the respiratory, digestive, endocrine, and muscular systems of mice

Jasmine Candeliere [1], Rebecca Krnel [1], Nicholas Laflamme [1], Darius Stamatakos [1]

[1] University of Ottawa, Ottawa, Ontario, Canada K1N 6N5

Currently, very little scientific literature investigates the adverse health effects of pesticides found in marijuana. With the legalization of marijuana, regulation of pesticide usage must also be modified since these crops are not merely ingested, they are also smoked, creating new chemical compounds that are potentially harmful to the human body. That being said, research shows that the combustion of federally approved pesticides produces chemicals that are harmful. This study investigates the potential effects of the combustion products of fenvalerate when they are inhaled. By studying mice, we have investigated the potential effects of these products on the respiratory, digestive and muscular system, as well as the overall adverse health effects.

The effects of cannabidiol and tetrahydrocannabinol on bone density in postmenopausal osteoporotic mice models Aseel Ahmad [1], Randa Ahmad [1]

[1] University of Ottawa, Ottawa, Ontario, Canada K1N 6N5

With the legalization of cannabis in Canada, many adults are now using it for self-treatment of various medical conditions. Cannabinoids such as THC and CBD, are capable of binding to various cell receptors in the body and alter how cells function and communicate. They can have therapeutic effects on numerous diseases, including epilepsy, neurodegenerative disorders, cardiovascular disorders, cancer, and osteoporosis. Osteoporosis is a degenerative disease, primarily affecting postmenopausal women - which causes a decline in bone density resulting in increased bone fragility. Considering the abundant amount of research indicating that components of cannabis, THC, and CBD, are capable of stimulating bone formation, this application requests the investigation of their effects on postmenopausal osteoporosis. We propose a randomized controlled trial using ovariectomized mouse models to simulate estrogen decline after menopause, resulting in osteoporosis. The models will be 
UNDERGRADUATE RESEARCH IN NATURAL AND CLINICAL SCIENCE AND TECHNOLOGY (URNCST) JOURNAL Read more URNCST Journal articles and submit your own today at: https://www.urncst.com

divided to undergo treatments of THC, CBD, equal combination, and placebo. Bone densities before and after treatments are to be analyzed using DXA method of scanning.

Introduction of gamma-aminobutyric acid into the bloodstream to negate NMDA receptor hypofunction induced by $\Delta$ 9-THC

Jebriel Abdul [1], Max Yan [1], Maxwell Zeggil [1]

[1] University of Ottawa, Ottawa, Ontario, Canada K1N 6N5

Emergent evidence relating to the inhibition of long-term potentiation by THC activating CB1 receptors and inhibiting the ability to stop production of glutamate. An excess of Glutamate will activate the postsynaptic NMDA and AMPA receptors in the neurons most commonly found on the hippocampus and surrounding regions. This contributes to many of the feelings of being "high". However, in heavy and habitual users of cannabis this process does not allow for the long-term potentiation in these synapses which affects memory, learning, reasoning and other aspects of one's function. These same losses of LTP can be seen in people with schizophrenia and neurodegenerative disorders and happen to all of us as we age. There are certain substances which can bind to the CB1 receptor and stop the THC from binding to the CB1 receptors as effectively. The GAD67 molecule, which we intend to use to achieve this and lower the harmful effects of THC on the brain, will be distributed to mice in this experiment and the behaviour of the mice will be monitored. THC affected, THC and GAD67 affected, and normal mice will be subjected to tests which will show differences in their learning, spatial awareness and orientation, and reasoning abilities.

Using activated charcoal filters to deactivate pesticides found in cannabis smoke reducing harmful effects to consumers Réale El-Hayek [1], Jessica Elkesserwani [1], Gabrielle Lalonde-Leblond [1]

[1] University of Ottawa, Ottawa, Ontario, Canada K1N 6N5

Pesticides found in cannabis smoke can cause great harm to consumers. We hypothesized that an activated charcoal filter would neutralize the effects of pesticides, rendering the practice safer. Through lab-based research, we studied the health hazards related to pesticides contained in marijuana. We then studied the effectiveness of a filter. The method consisted of experiments conducted on 64 male rats divided into eight groups (control (1)), cannabis without pesticides (2), pesticide only (3), three groups with three different concentrations of the pesticide on cannabis (4, 5 and 6), and cannabis with pesticide deactivated by the activated charcoal filter ( 7 and 8)). This allows for the evaluation of the effect of three concentrations of a legally-approved pesticide and the effect of the activated charcoal filter. After 24 weeks of experimentation, we confirmed which dose of pesticide causes significant harm, as well as the effectiveness of the activated charcoal filter.

The development of a novel MAGL-activated CB1R antagonist prodrug to reduce negative long-term effects of THC on the pre-adult brain

Samuel Benzaquen [1], Joseph Ostrovsky [1]

[1] University of Ottawa, Ottawa, Ontario, Canada K1N 6N5

Chemicals within cannabis can have negative effects on developing neurons, affecting the brain structures of fetuses, children, and adolescents, resulting in disadvantages in terms of cognition, brain development, and memory. The structural difference in developing brains with and without cannabis exposure points to cannabis phytocannabinoid disruption of the regulatory endocannabinoid system that guides neuron development. In a developing neuron, there is an enzyme gradient of monoacylglycerol lipase (MAGL) along the axon, decreasing towards the growth cone. MAGL regulates neural growth by degrading the endocannabinoid 2-arachidonoylglycerol, which binds to CB1 and downregulates SCG10, a stathmin that increases microtubule instability. The phytocannabinoid tetrahydrocannabinol (THC) present in cannabis, however, is not degraded by MAGL, and thus, when ingested, THC hyperactivates CB1 receptors, and downregulates SCG10 excessively, resulting in over stability of neural microtubules. This results in ectopic neural connections and growth that impede the proper development of brain structures and function. In order to counteract this activity in a selective way that does not interfere with proper cell function, this study proposes first an examination of the CB1R antagonist activity of the phytocannabinoid precursor tetrahydrocannabivaric acid (THCVA), and the synthesis and examination of the anticannabinoid properties of a monoacylglycerol prodrug 2-tetrahydrocannabivarinacetoxyglycerol (2-THCVA-G). When metabolized by MAGL, 2THCVA-G releases THCVA. Since MAGL exists in a gradient along the axon, THCVA can be selectively activated in areas where CB1R antagonism is required, inhibiting THC hyperactivation without excessively affecting both the normal growth of the neuron and the psychoactive properties of THC.

Cyr et al. | URNCST Journal (2019): Volume 3, Issue 9

Page A11 of A19

DOI Link: https://doi.org/10.26685/urnest.163 
UNDERGRADUATE RESEARCH IN NATURAL AND CLINICAL SCIENCE AND TECHNOLOGY (URNCST) JOURNAL Read more URNCST Journal articles and submit your own today at: https://www.urncst.com

\author{
Applying the anti-oxidative effects of cannabidiol to inhibit overexpressed calcium modulated kinase II in heart failure \\ phenotypes to attenuate calcium leaking and improve contractility \\ Anna Boukina [1], Madiha Sadaf [1], Layla Ziha [1] \\ [1] University of Ottawa, Ottawa, Ontario, Canada K1N 6N5
}

Current research of cannabidiol's (CBD) antioxidant properties do not focus on the heart. However, many applications can be used to test how its anti-oxidizing properties can help reduce heart rhythm abnormalities. This experiment investigates the use of CBD to interfere with reactive oxygen species $\mathrm{H} 2 \mathrm{O} 2$, to prevent the over-activation of $\mathrm{Ca} 2+/$ calmodulin dependent protein kinase (CaMKII $)$.

Prenatal cannabidiol administration on mice growth and development: A preliminary study for use during human labour

Catherine Everest [1], Alexandra Goudreau [1], Emily Valko [1]

[1] University of Ottawa, Ottawa, Ontario, Canada K1N 6N5

Uterine contractions are a painful but expected byproduct of childbirth, however, traditional pain management such as epidurals have the possibility of negative side effects for both mother and child. Cannabidiol (CBD), a non-psychoactive compound derived from cannabis, has indications for medicinal potential with pain management, as it binds to serotonin receptors which control pain sensation. Using an animal model, we aim to test the effect maternal CBD has on the growth and development of the offspring by histological brain examination and behavioural testing of mice. In addition, we will perform human work to determine CBD's ability to cross the placenta by human placental perfusion. It is believed that moderate concentrations of CBD will cross the placenta after acute maternal administration, and there will be no difference in brain development nor behaviour of mice pups.

Screening of cannabis components' involvement in DNA breakage and epigenetic changes Rim Al-Siss [1], Mariam Taktek [1], Si Yu Zhang [1]

[1] University of Ottawa, Ottawa, Ontario, Canada K1N 6N5

Cannabis and its components have been extensively studied for their therapeutic uses such as in the treatment of epilepsy, chronic pain, loss of appetite, etc. However, there is concern that long-term use of cannabis can cause genetic changes. Studies have shown a correlation between cannabis use and DNA aberrations as well as cancer-induction. However, it is unclear that this correlation is equal to causation. Our study aims to understand the effects of the major cannabis components on DNA, in particular, DNA breakage and epigenetic changes which may lead to cancer development. Cannabis components, such as Tetrahydrocannabinol (THC), Cannabidiol (CBD), Tetrahydrocannabinolic Acid (THCA), Cannabinol (CBN) and Cannabichromene (CBC) will first be screened to evaluate their genotoxicity through a Comet assay. Their potential effect on gene expression will then be assessed by a combined RNA microarray assay and a Serial Analysis of Gene Expression (SAGE), followed-up by a targeted Chromatin Immunoprecipitation (ChiP) assay.

Identifying the repellent genes in cannabis $(C$. sativa) through CRISPR screening: The hidden use of marijuana Shawna Kim [1], Ambre Lambert [1], Rachel Miller [1], Anmol Saxena [1]

[1] Queen's University, Kingston, Ontario, Canada K7L 3N6

Chemical pesticides have caused numerous deaths of people, animals, and plants. As a result, alternative pesticides which are health beneficial and ecological are needed. Cannabis sativa, known for its psychoactive effects, can be the solution to this problem. It has excellent repellent characteristics as seen through its use as a companion plant, as well as in-vitro studies. However, it has its drawbacks due its controversial nature and lack of research. To solve this problem, our paper aims to locate the non-vital genes in $C$. sativa that cause its repellent effects (R-genes) through CRISPR screening. To optimally identify the R-genes, the random knocked out genes of $C$. sativa were compared to the percentage of alive root-knot nematodes ( $M$. incognita) in the plant's soil. In our experiment, four plants were established per sample: Plant A which is a normal Cannabis sativa, Plant B which is a normal Cannabis sativa being infected by $M$. incognita, Plant $\mathrm{C}$ which is a genetically modified Cannabis sativa, and Plant D which is the same as Plant C except it is being infected by $M$. incognita. Then the percentage of alive nematodes will be compared in Plant B and D to identify the R genes. The discovery of R-genes is important as it can be

Cyr et al. | URNCST Journal (2019): Volume 3, Issue 9

Page A12 of A19

DOI Link: https://doi.org/10.26685/urnest.163 
UNDERGRADUATE RESEARCH IN NATURAL AND CLINICAL SCIENCE AND TECHNOLOGY (URNCST) JOURNAL Read more URNCST Journal articles and submit your own today at: https://www.urncst.com

used to discover a new class of repellent molecules. They can also be inserted into crops or household plants, giving them Cannabis sativa's repellent effects, and benefiting agricultural and health fields.

\author{
The use of cannabis to taper off opioid medication in opioid-dependent chronic pain patients \\ Tashifa Imtiaz [1] \\ [1] Queen's University, Kingston, Ontario, Canada K7L 3N6
}

Chronic pain affects approximately 1 in 5 Canadians and is the leading cause of disability among working-age adults. Opioids have become a more common pharmacological intervention in the treatment of non-cancerous chronic pain in Canada with daily dose dispensing tripling between 2000 and 2014. As opioid-dispensing increases so has opioid-related deaths; 4000 Canadians died of opioid overdose in 2017. Opioid tapering, involving slowly decreasing patients' opioid dosage, has been proposed as an intervention to address opioid-dependence in chronic pain patients. Cannabis has been shown to produce similar analgesic effects to opioids. Cannabis may also work synergistically with opioids and can augment their analgesic effects. Furthermore, cannabis has been shown to decrease drug-seeking behaviour. This research suggests strong potential for cannabis to address chronic pain and mitigate the risk of opioid overdose in opioid-dependent chronic pain patients. We propose a clinical trial to measure the effectiveness of a tapering intervention that incorporates cannabis. The clinical trial will compare tapering with cannabis to tapering without cannabis in opioid-dependent chronic pain patients identified through a family health care team in Kingston, Ontario. A triple-blind procedure will be used so that the patients, drug dispensers and outcome assessors will not have knowledge of which patients have been given cannabis. Three outcomes will be measured: chronic pain using a method outlined by the Initiative on Methods, Measurement, and Pain Assessment in Clinical Trials (IMMPACT), drug-seeking behaviour assessed with a survey and overall satisfaction with course of intervention measured using a survey.

\author{
A characterization of the underlying regulatory mechanism of CB1R - 5-HT2AR heterodimerization \\ Rania Belhadjhamida [1], Eesha Lodhi [1], Mustafa Sherik [1] \\ [1] Queen's University, Kingston, Ontario, Canada K7L 3N6
}

Activation of type 1 cannabinoid receptors (CB1R) by $\Delta 9$-tetrahydrocannabinol (THC) provides the behavioural outcomes of analgesia, anti-anxiety and neural protection. While beneficial for use as a therapeutic agent, THC also produces a variety of negative effects with major consequences such as memory deficit. As a result, there is large interest in isolating and harnessing the beneficial effects of THC. Behavioural studies carried out in 5-HT2A knock-out mice revealed that the specific effects of memory deficit, and anxiolytic effects induced by THC were under the control of 5-HT2A only when it was hetero-dimerized to $\mathrm{CB} 1 \mathrm{R}$ in the hippocampus and prefrontal cortex. While previous literature has been able to depict the roles of each receptor upon dimer formation, the mechanisms of regulation and induction of dimerization remain unknown. By analyzing assessments of regulatory mechanisms of G-protein coupled receptor hetero- and homo-dimers of previous literature, our goal is devising our own method of characterizing the regulatory mechanism. This will be done using a multi-time point study that assesses for dimerization using FRET and quantification experiments within the time frame between biosynthesis of the respective receptors at the endoplasmic reticulum up to incorporation into the plasma membrane and interaction with the THC agonist. The time in which dimerization is identified will provide insight into the specific location and portion of the biosynthesis process from which further assessment of the regulatory mechanism can be conducted.

Ingestion versus inhalation: Effect of method of marijuana consumption on brain activity Yusef Khan [1], Sulayman Syed [1], Titobi Wuraola [1], Shahroze Zafar [1]

[1] University of Toronto Scarborough, Scarborough, Ontario, Canada M1C 1A4

In Canada's current climate of marijuana legalization, many questions are raised about marijuana usage and its subsequent effect on users and how the effect can be quantified. Marijuana can be utilized through many different ways, in multiple different forms and strains. What this study aims to do is to understand the ramifications associated with the different forms of marijuana available. This study aims to explore the varying effects that different forms of marijuana intake entail and understand what the different forms affect in a clinical setting through the usage of fMRI. The three different forms of marijuana intake that are explored by this study are; through a joint, through a water bong, and through the use of edibles. The individual will go through a rigorous interview process in the fMRI to effectively map the differing effects that the different forms of intake may have. The amount of specific chemicals found in marijuana, mainly THC and CBD, are to be closely administered and it will be ensured that each individual has only $5 \mathrm{mg}$ of each.

Cyr et al. | URNCST Journal (2019): Volume 3, Issue 9

Page A13 of A19

DOI Link: https://doi.org/10.26685/urnest.163 
UNDERGRADUATE RESEARCH IN NATURAL AND CLINICAL SCIENCE AND TECHNOLOGY (URNCST) JOURNAL Read more URNCST Journal articles and submit your own today at: https://www.urncst.com

\author{
Hungry and high: Effects of malnutrition on motor functioning \\ Girima Bhalla [1], Ilakkiah Chandran [1], Nivetha Chandran [1], Jennifer Gong [1] \\ [1] University Of Toronto Scarborough, Scarborough, Ontario, Canada MIC 1A4
}

\begin{abstract}
Although cannabis has officially been legalized in Canada and some states in the United States, there is still controversy due to the impacts of consumption on the mental functioning of consumers. It is notable that the frontal lobes are greatly impacted by cannabis use and activity within this region decreases when it is paired with insufficient meal consumption. This is due to the fact that the endocannabinoids in cannabis tend to increase appetite. Those who live in food deserts are facing a greater burden as they lack healthy food options. This study focuses on the ways in which cannabis consumption, paired with meals of varying nutritional content, enhances activation in endocannabinoid receptors and the mesolimbic pathway, resulting in changes within the motor cortex. It is expected that the motor cortex will be impaired by cannabis consumption and that poor nutritional content will lead to increased impairment. Mice will be divided into two groups, only one of which will consume cannabis, and they will be fed diets that vary in nutritional content. They will enter the Tolman maze while simultaneously being assessed for motor cortex activity. It is anticipated that activity in this region will decline and levels of dopamine and anandamide will increase, signifying impairment. The results of this study will provide insight into the interaction between the social determinants of health and cannabis consumption, specifically in terms of cognitive functioning amongst those that reside in food deserts.
\end{abstract}

Investigating the effect of THC on BDNF levels in depressed male rats Said Aoude [1], Jenan Bouakkar [1], Sebastian Dowhanik [1], Hafsah Sheikh [1]

[1] University of Toronto Scarborough, Scarborough, Ontario, Canada M1C 1A4

Despite the legalization of cannabis in Canada, researchers have yet to understand the extent of its side effects on neural development. This is especially true when considering the impact of cannabis on those with mental illnesses including depression. One vital protein involved in the growth and differentiation of neurons in the brain is the brain-derived neurotrophic factor (BDNF). The aim of our proposed study is to evaluate how the neural health of those suffering from depression is impacted by $\Delta 9$-tetrahydrocannabinol (THC) consumption, the primary psychoactive component of cannabis, in comparison to those without depression by comparing their respective BDNF levels. To test this, we will use olfactory bulbectomised rats as models for depression and measure the BDNF concentration in their frontal cortices, hippocampi, and amygdalae following exposure to THC. BDNF will be detected via Western blot and quantified using BDNF-specific ELISAs. Our study could influence how society views the risks associated with the use of cannabis amongst depressed individuals.

THC-mediated inhibition of acetylcholinesterase as a novel therapeutic mechanism for managing Alzheimer's disease progression and symptomatology Samuel Haile [1], Abhay Issar [1], Anshu Kashyap [1], Raza Syed [1]

[1] University of Toronto Scarborough, Scarborough, Ontario, Canada MIC 1A4

Alzheimer's disease (AD) is a chronic neurodegenerative disease characterized by the progressive deterioration of cognitive function and is widely hypothesized to be driven by the production and deposition of $\beta$-amyloid plaques. Since AD primarily affects older populations, Canada's rapidly aging population presents the salient issue of mitigating this emerging public health crisis. Currently, there are only four Health Canada approved drugs for the treatment of the symptoms of AD, three of which are acetylcholinesterase (AChE) inhibitors. The recent legalization of cannabis, and trans- $\Delta 9$-tetrahydrocannabinol (THC) by extension, has greatly increased research interest in THC as a potential therapy for both AD symptoms and progression due to its similar function as an AChE inhibitor. The longstanding status of cannabis as a controlled substance has contributed to the absence of literature examining its therapeutic capabilities and thus, we offer a novel therapeutic mechanism through which THC manages the symptoms and slows the progression of AD pathology. We hypothesize that THC will enhance cognitive functions through its role as an $\mathrm{AChE}$ inhibitor, reducing turnover of the acetylcholine neurotransmitter that holds a central role in learning and memory--processes known to be impaired in AD. We propose a study that will assess these effects using $\beta$-amyloid precursor protein transgenic mouse lines that will be exposed to a saline solution (control), THC, and Galantaminea Health Canada approved AChE inhibitor. Behavioural tasks and biochemical assays will be utilised to measure the hypothesized effects of THC on AD pathology.

Cyr et al. | URNCST Journal (2019): Volume 3, Issue 9

Page A14 of A19

DOI Link: https://doi.org/10.26685/urnest.163 
UNDERGRADUATE RESEARCH IN NATURAL AND CLINICAL SCIENCE AND TECHNOLOGY (URNCST) JOURNAL Read more URNCST Journal articles and submit your own today at: https://www.urncst.com

Developmental cannabis exposure on cognitive flexibility and neuron/brain morphology changes in the prefrontal cortex

Jonathan Chung [1], Anthony Nguyen [1], Lucas Omazic [1]

[1] University of Guelph, Guelph, Ontario, Canada N1G 2W1

Cannabis is one of the most commonly used substances during pregnancy. The components of this drug are able to cross the placental barrier and are transferred during lactation. Furthermore, perinatal cannabis exposure within the second and third trimesters of pregnancy have been associated longitudinally with cognitive deficit and volumetric decreases of cortical gray matter in the brain. Due to these correlations, we hypothesize that developmental cannabis effects cognitive function in the offspring of pregnant mice. We predict that cannabis exposure during the second/third trimester of pregnancy leads to decreased cognitive flexibility, reduced cortical gray matter and prefrontal cortex activity, and altered neuronal morphology. We propose to test these hypotheses in the offspring of 25 C57BL/6 cannabis-exposed pregnant mice by researching: 1) Exposure during certain trimesters of pregnancy on cognitive flexibility using a visual discrimination with reversal task (VDR). 2) Longitudinal effects on volume of cortical gray matter and activity of prefrontal cortex using functional Magnetic Resonance Imaging (fMRI) during VDR. 3) Neuronal structure changes using the Golgi-Cox Staining method. These outcomes are important to understand developmental cannabis exposure on the neurobiology of the brain. Furthermore, the longitudinal response is an important consideration as implications from perinatal substance abuse do not always present themselves immediately following birth. This information can be used to develop therapeutic interventions in affected children.

Proposed relationship between concentration of THC from passive cannabis consumption and episodic memory depletion

Rachel Cardey [1], Leah Horlings [1], Rachael Vella [1]

[1] University of Guelph, Guelph, Ontario, Canada N1G 2W1

In the midst of cannabis legalization in Canada, there arises a need to further our knowledge of its effects on the human body. Previous research has proven that second hand smoke from tobacco cigarettes have adverse health effects (Centers for Disease Control and Prevention, n.d.). Therefore, an interest emerges concerning the existence of a similar relationship between passive and active consumption of cannabis. With background from scientific research, we propose that concentrations of THC appear in the body from passive cannabis consumption. We believe these concentrations can then have negative implications on cognitive processes, specifically verbal episodic memory. If proven to have an effect, regulation regarding areas permitted for smoking of cannabis becomes important as unwanted effects could be seen in those unintentionally consuming it. Our proposed research experiment involves comparing THC levels and episodic memory between passive and active cannabis users.

The effects of prolonged exposure to THC during early adulthood on long- term sub-cortical ventral tegmental area dopaminergic firing and anxiogenic characteristics in male rats Claudia Lutelmowski [1], Stefanie Stockhamer [1], Danna Ellner Yerushalmi [1]

[1] University of Guelph, Guelph, Ontario, Canada N1G 2W1

Since legalization of marijuana, the minimum age for growth, purchase and consumption of cannabis in Canada is 18 or 19 years old (depending on province), which lies within a critical period of brain maturation. If the goal of legislators is to promote public health, they are not protecting the cohort of individuals between 18-25 years old, as their brains are incredibly vulnerable to consequences of cannabis use 2-5. This study aims to determine early adulthood brain development by observing the long term consequences of $\Delta 9$-tetrahydrocannabinol (THC) exposure during three different life stages in rats. We will use electrophysiological recordings and the Light Dark Box Test to measure the dopaminergic activity in the ventral tegmental area (VTA) and anxiogenic characteristics when vaporized THC is administered during adolescence, early adulthood and later adulthood. Results from Renard et al. (2017) show that THC administration to adolescents increases dopaminergic activity in the VTA as well as increases anxiogenic activity in the long term 6 . The study determined that THC exposure to adults caused no change, but did not test exposure to young adults 6 . It is predicted that outcomes for young adult rats will be more closely related to adolescent rats than their adult counterparts. The results will exemplify the importance of studying young adult brain development, which is largely overlooked in animal research, and emphasizes susceptibility to long-term consequences from chronic cannabis use between 18-25-year old's. This study aims to educate legislators and young adults regarding the unique consequences of consuming marijuana during this age.

Cyr et al. | URNCST Journal (2019): Volume 3, Issue 9

Page A15 of A19

DOI Link: https://doi.org/10.26685/urnest.163 
UNDERGRADUATE RESEARCH IN NATURAL AND CLINICAL SCIENCE AND TECHNOLOGY (URNCST) JOURNAL Read more URNCST Journal articles and submit your own today at: https://www.urncst.com

Cannabidiol as a potential treatment for preeclampsia

Madison Best [1], Hesham Farag [1], Rachel Wilkes [1], Nicholas Zingone [1]

[1] University of Guelph, Guelph, Ontario, Canada N1G 2W1

Preeclampsia $(\mathrm{PE})$ is a common pregnancy complication that leaves the affected individual to choose between preterm delivery or risking death. These outcomes are far from ideal and the search for a better treatment is underway. Due to preliminary studies implicating Cannabis use as a risk factor for the development of PE as well as other partum complications, it has yet to be investigated as a treatment method. With so many studies showing Cannabis as a treatment method for a variety of the most dangerous symptoms of PE, it may be possible that Cannabis will allow future mothers afflicted with PE to bring their child to full-term. In this study novel mouse models of PE will be utilized to demonstrate the effect of cannabidiol on expecting mothers who are predisposed to PE.

Investigating the effect of varying THC to CBD ratios on cognition Estelle Tang [1], Jeffrey To [1]

[1] University of Toronto St. George, Toronto, Ontario, Canada M5S

Recent literature shows a potentially protective effect of cannabidiol (CBD) to alleviate cognitive-impairing symptoms of tetrahydrocannabinol (THC). The endocannabinoid system, composed of endocannabinoid receptors, endocannabinoids, and metabolic enzymes, is highly involved in emotional and behavioral regulation due to the fact that activating CB1 inhibits excessive activity of neurotransmitters such as dopamine, serotonin, and endogenous opioids. CBD has the potential even in minimal quantities to inhibit the consequential effects of THC. CBD itself has no visible effects until the EC system becomes unbalanced by a stimulus, such as pain, or additional cannabinoid, such as THC. In order to reap the most benefits, the ratio of THC/CBD and dose administration timing is crucial. This experiment will use a between-subjects design to test working memory after varying THC to CBD ratios.

The combined psychophysiological effects of THC and CBD manifesting in the suppression of nociception in mice Fang-Chi Chang [1], Qirui Ding [1], Stephanie Tam [1], Ruiyang Xu [1]

[1] University of Toronto St. George, Toronto, Ontario, Canada M5S

With the national legalization of cannabis, there is greater need for scientific research to ensure the public is informed on all aspects regarding its use. While its psychotropic properties are an essential part of its appeal, there exists many other effects of lesser recreational importance that, nonetheless, need to be further addressed. Its analgesic effects specifically are already a target in the development of medical cannabis for the treatment of pain and associated diseases; however, they are often undermentioned outside of medicine. We aim to investigate the extent to which the combined psychological effects of THC and CBD manifest in the suppression of nociception. With the use of mouse models and the peripheral pain inducer, URB937, experimentation will be conducted through the introduction of formalin following cannabis vaporization to assess pain response and determine possible correlations with the psychological effects of THC. Results from this experiment have the potential to generate prospects for future studies in regards to the diverse properties of cannabis.

Cannabis and the gut microbiota: Impact on obesity

Jim Chen [1], Abdullah Malik [1], Helen Yang [1], Frederick Zhang [1]

[1] University of Toronto St. George, Toronto, Ontario, Canada M5S

Cannabis is now legal in Canada for recreational use. In contrast to well-characterized drugs like alcohol, chronic cannabis use has unknown implications on human physiology. For example, chronic alcohol use has been linked to obesity and weight gain, while cannabis has not. While chronic use of cannabis increases feeding behaviour, a paradoxical negative correlation between cannabis use and obesity has been observed. Previous studies have highlighted the potential use of cannabis as a therapeutic to reduce weight gain, although the mechanism remains unclear. An area of particular interest is the effect of cannabis on the gut microbiome. Modulation of the gut microbiome is becoming increasingly important to understand existing and proposed mechanisms of metabolic diseases such as obesity. This study aims to characterize changes to the gut microbiome after regular exposure to $\Delta 9$-tetrahydrocannabinol (THC), a primary cannabinoid, in mouse models. Identifying changes to the gut microbiome, through functional metagenomics approaches, will supplement our current understanding of cannabinoids and 
UNDERGRADUATE RESEARCH IN NATURAL AND CLINICAL SCIENCE AND TECHNOLOGY (URNCST) JOURNAL Read more URNCST Journal articles and submit your own today at: https://www.urncst.com

energy homeostasis. Avoiding the major pitfalls of previous attempts with cannabis as a therapeutic, this study aims to use specific changes to the gut microbiome to direct future therapeutic developments.

Cannabis consumption in Nrg1-HET individuals poses a significant risk factor for schizophrenia-like symptom development

Mansour Dehan [1], Yara El Bardisi [1], Luke Pham [1], Di Wu [1]

[1] University of Toronto St. George, Toronto, Ontario, Canada M5S

Neuregulin 1 (Nrg1) and cannabis consumption have long been implicated as potential risk factors for developing schizophrenia (Sz). Previous studies have been able to determine correlational links between Nrg1-Sz and Cannabis-Sz, but haven't been able to create a working model of Nrg1-Cannabis interactions and their relation to Sz development. Some background information is available on the effect of Nrg1 on the cannabinoid system; specifically, the modulation of Nrg1 on cannabinoid receptor type 1 (CB1), resulting in cannabis-mediated information processing dysfunction and an increased risk of cannabis dependence. This study aims to provide a working model of how Nrg1-Cannabis interactions in the cortex contribute to the development of certain schizophrenic symptoms. We hypothesize that Nrg1-Cannabis interactions in the hippocampus alter N-methyl-Daspartate receptor (NMDA receptors) expression, and thus induces changes in the mesocorticolimbic system resulting in typical negative and cognitive symptoms of Sz. These include poor memory, increased reward-seeking behaviour, and reduced sociability. We aim to create an animal model using transgenic Nrg1-HET mice, which we can then manipulate to demonstrate the causal relationship between Nrg1-Cannabis interactions and the development of Sz-like symptoms.

Turning up the heat on glioblastoma multiforme: Heat shock protein inhibitors to enhance the anti-tumour effects of cannabidiol in temozolomide and cannabinoid combination therapy

Amna Ahmed [1], Amna Majeed [1], Teresa Zhu [1]

[1] University of Toronto St. George, Toronto, Ontario, Canada M5S

Glioblastoma multiforme (GBM) is the most common and aggressive type of glioma, known to be highly resistant to treatment. Research thus far has focused on developing more effective therapies, including those targeting the endocannabinoid system (ECS). With the legalization of marijuana, research is increasingly interested in the anti-tumour effects of the psychoactive $\Delta 9$ tetrahydrocannabinol (THC) and the non-psychoactive cannabidiol (CBD), phytocannabinoids derived from the plant Cannabis sativa. Notably, temozolomide (TMZ), the benchmark chemotherapeutic agent for GBM, has demonstrated greater cytotoxicity when combined with THC and CBD. CBD in particular is thought to induce apoptosis through an increase in reactive oxygen species (ROS). Our proposal aims to enhance the anti-tumour effects of CBD through inhibiting heat shock proteins (HSPs), which protect the cell from elevated ROS. Varying ratios of THC:CBD will be applied in conjunction with TMZ and HSP inhibitors to murine models of GBM and tumour cell viability measured. This research will further optimize cannabinoid-based drug therapies for GBM, moving towards a model with increased cytotoxicity and decreased psychoactivity.

Detection of cannabis in saliva and determination of corresponding THC blood concentrations Alina He [1], Marina Nysten [1], Farida Rahman [1], Joyce Wu [1] [1] McGill University, Montreal, Quebec, Canada H3A OG4

Cannabis impairs driving by affecting cognitive and psychomotor performances. These impairments are caused by $\Delta 9$ tetrahydrocannabinol (THC), which is the main psychoactive ingredient in cannabis. With the recent legalization of cannabis in Canada, there is a need for a reliable roadside test that can identify cannabis impaired drivers in a simple and efficient manner. Saliva is well suited for roadside detection and blood tests are reliable for testing THC concentration. Traces of THC in saliva samples can be detected using azo dyes, a diverse class of coloured molecules. We propose to create a scale correlating saliva test colour results to THC blood concentration. In order to develop a scale that can be used for a range of THC concentrations, mice will receive a specific dose of THC within the range of $0.2 \mathrm{mg} / \mathrm{Kg}$ to $100 \mathrm{mg} / \mathrm{Kg}$. Blood and saliva samples will be collected. Blood samples will be tested for THC concentration and a series of tests using azo dyes will be applied to saliva samples to produce colour changes corresponding to a specific range in concentration. The resulting colours can be mapped against the blood THC concentration to produce a scale. Despite the recent legalization of cannabis in Canada, current legislation and technology are not adequate to reliably and efficiently measure cannabis intoxication roadside. This field of scientific inquiry has the potential to improve road-side detection of cannabis-induced impaired driving and increase the safety of Canadian roads.

Cyr et al. | URNCST Journal (2019): Volume 3, Issue 9

Page A17 of A19

DOI Link: https://doi.org/10.26685/urnest.163 
UNDERGRADUATE RESEARCH IN NATURAL AND CLINICAL SCIENCE AND TECHNOLOGY (URNCST) JOURNAL Read more URNCST Journal articles and submit your own today at: https://www.urncst.com

Impact of cannabis compounds on microbial communities

Alex Deckert [1], Anthony DeSouza [1], Jessica Leger [1]

[1] University of Ontario Institute of Technology, Oshawa, Ontario, Canada L1G OC5

Cannabis production is escalating with legalization in Canada. Therefore, environmental contamination of waste products from this industry is an emerging issue. The impact of cannabis compost material on soil microbial communities has yet to be investigated. Soil samples taken from representative areas (agricultural fields, natural land, and landfill sites) will be subjected to $1: 3$ and $1: 2 \mathrm{w} / \mathrm{w}$ cannabis compost to soil, under anaerobic and aerobic conditions for 30 days. Species diversity, species richness, and relative abundance of bacteria will be determined for each sample, using qPCR and gene sequencing. Results will lead to making informed decisions for legislation of waste in the future.

Reduction of psychoactive components in marijuana via growth conditions: For epilepsy treatment Elshaimaa Abdella Abdella [1], Menna Abdella [2], Fahreen Bushra [3], Nishitha Parial [4]

[1] University of Toronto St. George, Toronto, Ontario, Canada M5S

[2] Ryerson University, Toronto, Ontario, Canada M5B $2 K 3$

[3] Western University, London, Ontario, Canada N6A $3 K 7$

[4] York University, Toronto, Ontario, Canada M3J 1 P3

Recent legalization of cannabis has made medical marijuana a more available option to those that may benefit from its medicinal properties. Non-psychoactive ingredients of cannabis have shown to be an effective treatment in controlling recurring seizures. Whereas psychoactive ingredients of cannabis have been shown to have adverse effects, often increasing the frequency of seizures in epileptic patients. For these reasons, non-psychoactive ingredients such as CBD are often isolated or artificially synthesized when used in medicine to treat epilepsy. However, this isolation reduces the synergistic positive effects when consuming the raw plant as a whole. Furthermore, it has been seen that growth conditions of a cannabis plant can have a significant role in the plant's morphology, and its accumulation of psychoactive and non-psychoactive ingredients. Taking these factors into account, we propose to naturally minimize psychoactive ingredients and maximize non-psychoactive ingredients of cannabis through growth conditions of the plant. Doing so, we hope to construct a procedure which can prepare whole cannabis plants to both be consumed by epileptic patients as well as retain its synergistic benefits.

\section{Conflicts of Interest}

The authors have no conflict of interest to declare.

\section{Authors' Contributions}

DC: Founder and Vice-President of the Undergraduate Research Initiative, served on the planning committee for the USCC, assisted authors with their abstract submissions, drafted the conference abstract booklet, and gave final approval of the version to be published.

JG: Founder and President of the Undergraduate Research Initiative, served on the planning committee for the USCC, drafted the conference abstract booklet, and gave final approval of the version to be published.

RR: Served as President of Scinapse and Chair of the USCC planning committee, assisted authors with their abstract submissions, drafted the conference abstract booklet and gave final approval of the version to be published.

\section{Acknowledgements}

We want to acknowledge the entire Scinapse Provincial Team for playing an integral role in making this year's USCC a big success. The team that helped make this competition possible includes: Rashida Rajgara as President and Chair of the planning committee, Jamie Ghossein as President of URI and USCC adviser, Céline Sayed and Dominic Cyr as VicePresidents of URI and USCC advisers, Sarah Houssari and Amira Rouabhi as communications directors, Dilpreet Bajwa and Amanda Vandewint as graphic designers, Alison Hamilton as mentorship lead, Wael Zhaghrini as operations team lead, Antony El-Khoury as project and statistics coordinator, Linda Mardiros as Provincial Coordinator, Justin Thomas and Justen Choueiry as uOttawa campus leaders, Émilie Bianco as Event manager and logistics coordinator, and Andréa Brabant as executive assistant.

The USCC would not have been successful without the contribution and work of the multiple different chapters of the Scinapse USCC situated across other universities in Ontario. 
UNDERGRADUATE RESEARCH IN NATURAL AND CLINICAL SCIENCE AND TECHNOLOGY (URNCST) JOURNAL Read more URNCST Journal articles and submit your own today at: https://www.urncst.com

We want to acknowledge all the professors and graduate students that participated as judges for the competition. Your valuable time and feedback to the participants has had a positive impact on their future success.

\section{Funding}

The Scinapse USCC is funded by the Undergraduate Research Initiative and contributions from the University of Ottawa, Faculty of Science and the Faculty of Medicine.

\section{Article Information}

Managing Editor: Jeremy Y. Ng

Article Dates: Received Sep 30 19; Published Oct 1819

\section{Citation}

Please cite this article as follows:

Cyr D, Ghossein J, Rajgara R. Scinapse 2018-2019 Undergraduate Science Case Competition: Cannabis, harm or health?

URNCST Journal. 2019 Oct 18: 3(9). https://urncst.com/index.php/urncst/article/view/163

DOI Link: https://doi.org/10.26685/urncst.163

\section{Copyright}

(C) Dominic Cyr, Jamie Ghossein, Rashida Rajgara. (2019). Published first in the Undergraduate Research in Natural and Clinical Science and Technology (URNCST) Journal. This is an open access article distributed under the terms of the Creative Commons Attribution License (https://creativecommons.org/licenses/by/4.0/), which permits unrestricted use, distribution, and reproduction in any medium, provided the original work, first published in the Undergraduate Research in Natural and Clinical Science and Technology (URNCST) Journal, is properly cited. The complete bibliographic information, a link to the original publication on http://www.urncst.com, as well as this copyright and license information must be included.

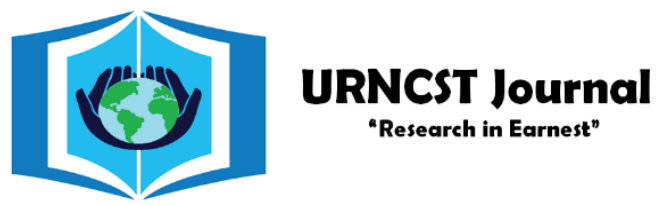

\section{Funded by the Government of Canada}

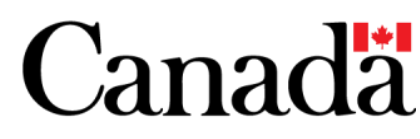

Do you research in earnest? Submit your next undergraduate research article to the URNCST Journal!

| Open Access | Peer-Reviewed | Rapid Turnaround Time | International |

| Broad and Multidisciplinary | Indexed | Innovative | Social Media Promoted |

Pre-submission inquiries? Send us an email at info@urncst.com | Facebook, Twitter and LinkedIn: @URNCST

Submit YOUR manuscript today at https://www.urncst.com!
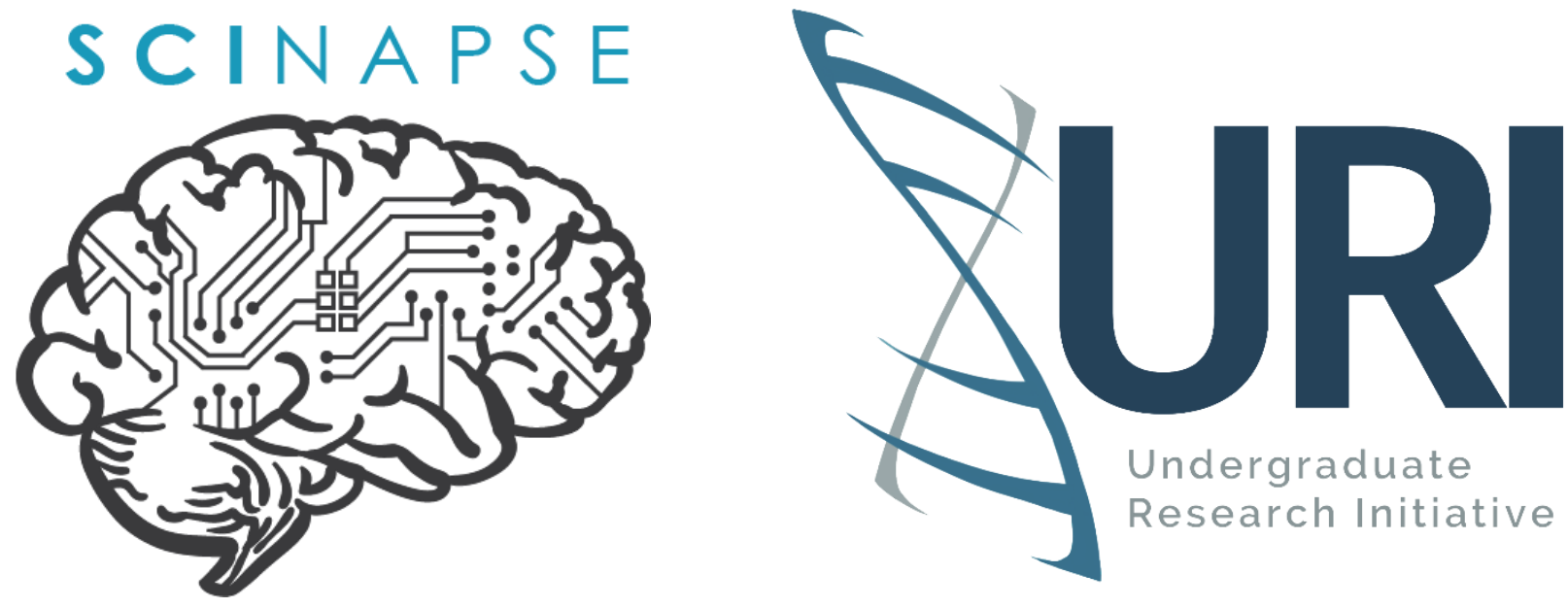

Cyr et al. | URNCST Journal (2019): Volume 3, Issue 9

DOI Link: https://doi.org/10.26685/urncst.163 